\title{
SCATTERING FROM LOADED GROOVES
}

\author{
Kasra Barkeshli \\ Department of Electrical Enginnering and Computer Science \\ The University of Michigan \\ Ann Arbor, Michigan 48109-2122 \\ Received September 9, 1991
}

\section{Abstract}

Electromagnetic scattering from a two-dimensional groove recessed in an arbitrarily thick conducting screen is studied. The groove may be empty or loaded with a lossy material which may or may not completely fill the cavity. For the partially loaded groove, the filling material is assumed electrically dense so that the standard impedance boundary condition is applicable at the top surface of the material. Employing a full-wave analysis, integral equations are derived for the tangential components of the electric field over the aperture. It is shown that the equations are identical for both partially loaded and completely loaded (or empty) cases provided that the aperture admittance of the groove is treated as the equivalent admittance of the internal medium looking into the aperture, thus simplifying the integral equations.

When the groove is completely filled by a dense material, the formulation reduces to that corresponding to a direct application of the impedance boundary condition over the aperture.

Key words: Electromagnetic scattering and detection, full-wave analysis, impedance boundary condition, cracks and grooves. 


\section{Introduction}

The study of electromagnetic scattering from filled cavities recessed in ground planes is important in many engineering applications because such cavities can be considered as approximate models for thin cracks in metal surfaces. At millimeter wave frequencies, electromagnetic scattering might be used in non-invasive detection and evaluation of gaps and cracks in structural systems for the purpose of failure prediction. Also, in radar signature analysis of various man-made structures, diffraction from cracks and joint openings can become a dominant mechanism in the bistatic scattering behavior of a target. Therefore, it is important to examine the effect of different geometries and material inserts on the scattering behavior of grooves.

When the operating wavelength, $\lambda_{o}$ and the aperture width of the groove, $w$ are such that the groove is electrically narrow $\left(w \ll \lambda_{o}\right)$, certain approximate solutions have been proposed. These include the transmission line model [1], the narrow resistive strip model based on Babinet's principle [2], and the closed from low-frequency solution [3]. In the latter, analytical expressions were derived for the equivalent magnetic current distribution and over the aperture of the narrow groove based on a quasi-static analysis of the exact integral equations.

In the millimeter wave region, however, the above narrow-width approximations are no longer valid and a more accurate solution is required. In this paper, an exact full-wave formulation is developed for the rectangular groove problem based on the Generalized Network Theory and the equivalence principle [4]. This theory has been applied to a number of related aperture and slot problems in the past $[5,6]$. In particular, we will extend the work of Auckland and Harrington who applied this theory to treat the TE transmission through a slit in a thick conducting screen. Employing the equivalence principle, the external fields are expressed in terms of the scattering integral while the fields internal to the cavity are given in terms of appropriate waveguide modes. An integral equation is then formed by enforcing continuity of tangential fields across the interface and is solved numerically via the method of moments. The problem will be formulated for general termination conditions as well as both principle polarizations.

When the groove is fully loaded by a lossy material, the analysis may be simplified by employing the impedance boundary condi- 
tion [7] and simulating the groove as an impedance insert. The type and complexity of the boundary condition used depend on the material composition. For high contrast material fillings, the standard impedance boundary condition (SIBC) yields good results [8] while higher order (generalized) boundary conditions (GIBC) may be more desirable for simulating material fillings of lower contrast [9].

The above studies treated the fully loaded cavities. For partially loaded grooves, on the other hand, the SIBC is still applicable at the top surface of the filling material provided the material can be assumed electrically dense. The range of validity for such an assumption will be discussed later. This renders a groove model with an imperfect termination and it is shown that the integral equations in this case are identical to those obtained for the completely loaded (or empty) case with an appropriate modification of the groove's aperture admittance.

\section{Full-Wave Formulation}

Consider the infinitely long groove of width $2 w$, and depth $d$ illuminated by the plane wave

$$
\mathbf{H}^{i}\left(\text { or } \mathbf{E}^{i}\right)=\widehat{z} e^{j k_{o}\left(x \cos \phi_{o}+y \sin \phi_{o}\right)}
$$

for H- (or E-) polarization, where $k_{o}=2 \pi / \lambda_{o}$ is the free space wave number and $\phi_{o}$ is the angle of incidence (Figure 1). The groove is

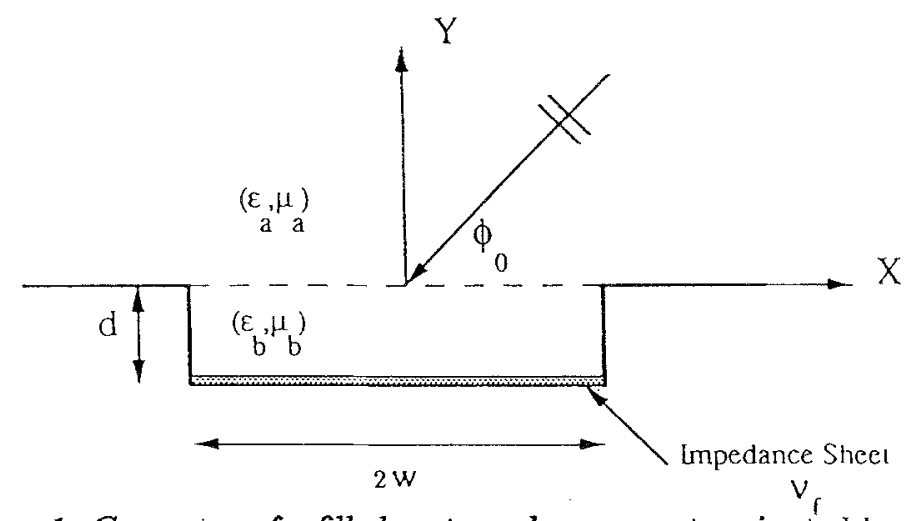

Figure 1: Geometry of a filled rectangular groove terminated by an impedance sheet. 
assumed to be filled with a lossy material of relative permittivity $\epsilon_{b}$, and permeability $\mu_{b}$, with an index of refraction $\mathcal{N}_{b}=\sqrt{\epsilon_{b} \mu_{b}}$ and terminated by an opaque sheet satisfying the impedance boundary condition

$$
\mathbf{H}-(\widehat{y} \cdot \mathbf{H}) \widehat{y}=v_{f} Y_{b} \mathbf{E} \times \widehat{y} \quad, \quad y=-d
$$

where $v_{f}$ is the normalized admittance at the groove's floor, $Y_{b}$ is the intrinsic impedance of the material filling the groove, and $(\mathbf{E}, \mathbf{H})$ are the fields at the top surface of the sheet. As will be shown later, this model can be used to simulate grooves partially loaded with electrically dense material. To formulate the scattered field by the groove, the Generalized Network Theory [4] is employed. Accordingly, the aperture is closed by a perfect conductor and the equivalent magnetic current

$$
\mathbf{M}=\mathbf{E} \times \widehat{n}=\mathbf{E} \times \widehat{y} \quad, \quad y=0
$$

is introduced over the aperture (equivalence principle, Figure 2). Here, $\mathbf{E}$ is the total electric field in the aperture of the groove. The scattered fields outside the cavity are those radiated by the equivalent magnetic current and consistent with the continuity of the tangential electric field across the aperture the field inside the cavity is that radiated by

$$
\mathbf{M}^{\prime}=\mathbf{E}^{-} \times \widehat{n}^{\prime}=-\mathbf{E}^{-} \times \widehat{n}=-\mathbf{E}^{+} \times \widehat{n}=-\mathbf{M}
$$

where $\mathbf{E}^{ \pm}$denote the total electric fields just above and below the aperture. To find the equivalent magnetic current we must also enforce the continuity of the tangential magnetic field across the aperture. We thus have

$$
\widehat{n} \times\left[\mathbf{H}^{a}(\mathbf{M})+\mathbf{H}^{i a}\right]=\widehat{n} \times \mathbf{H}^{b}\left(\mathbf{M}^{\prime}\right) \quad, \quad y=0
$$

where $\mathbf{H}^{i a}$ is the total field in the external region with the aperture closed, $\mathbf{H}^{a}$ represents the external scattered field, and $\mathbf{H}^{b}$ is the total field in the interior region.

To construct an integral equation in $\mathbf{M}, \mathbf{H}^{a}$ and $\mathbf{H}^{b}$ must be expressed in terms of the Green's function corresponding to each region. The tangential component of the external scattered field (attributed to $\mathbf{M}$ ) can be expressed as 


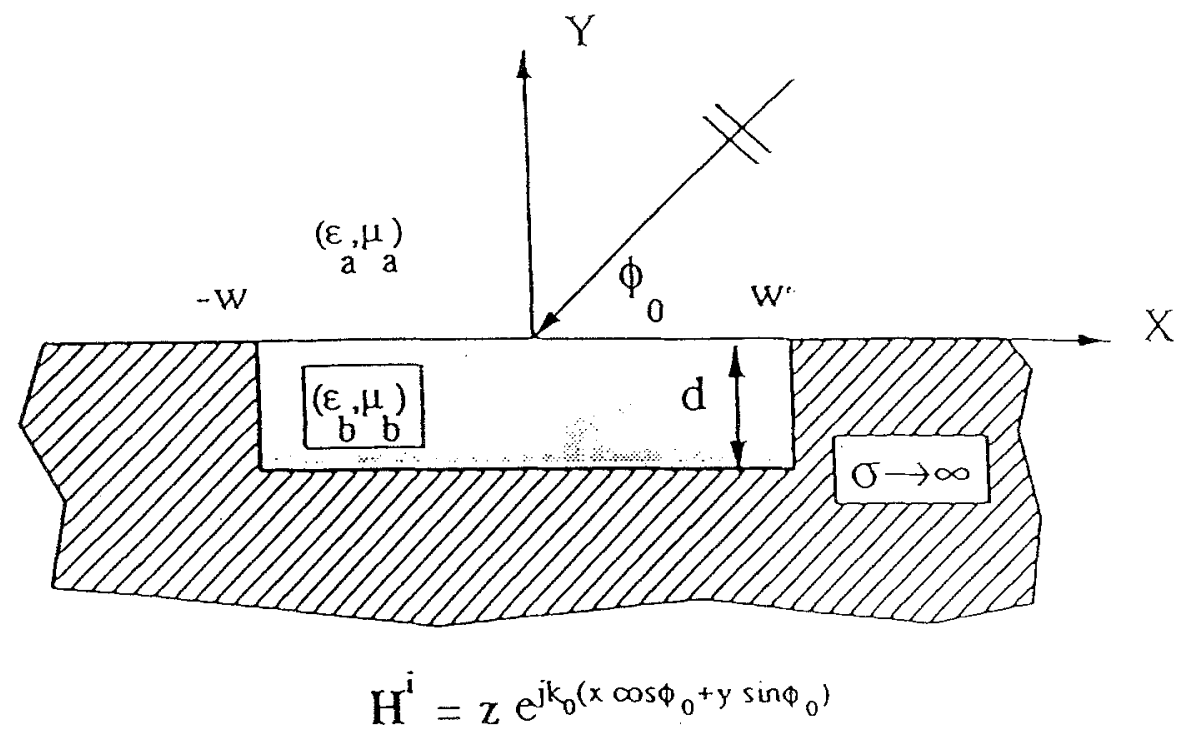

Equivalence Principle:

$\mathbf{M}=\mathbf{E} \times \mathbf{n}$

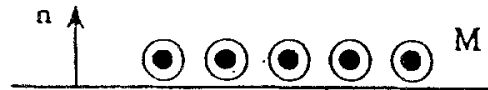

perfectly conducting

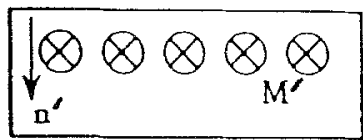

$M^{\prime}=E \times n^{\prime}=-E \times n=-M$
Continuity of Tangential E

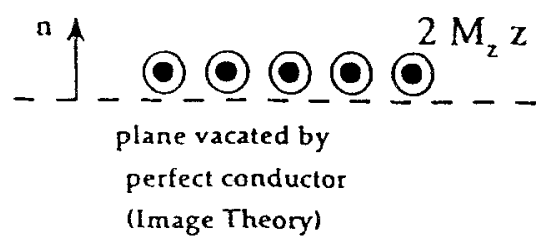

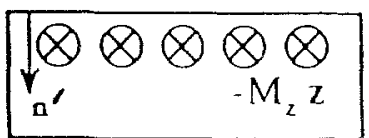

Figure 2: Application of the equivalence principle to the groove problem. 


$$
\mathbf{H}^{a}(\mathbf{r})=-j k_{0} Y_{0} \int_{-w}^{w} \mathbf{M}\left(x^{\prime}\right) \cdot \bar{\Gamma}\left(x ; x^{\prime}\right) d x^{\prime}
$$

where $Y_{o}$ is the intrinsic admittance of the free space and $\bar{\Gamma}$ is the half space dyadic Green's function

$$
\bar{\Gamma}\left(x ; x^{\prime}\right)=\frac{1}{2 j}\left[\left(1+\frac{1}{k_{o}^{2}} \frac{\partial^{2}}{\partial x^{2}}\right) \hat{x} \widehat{x}+\widehat{z} \hat{z}\right] H_{o}^{(2)}\left(k_{o}\left|x-x^{\prime}\right|\right)
$$

The internal fields (those attributed to $\mathbf{M}^{\prime}$ ) can be written in terms of the $\mathrm{TM}_{\mathrm{Z}}$ and $\mathrm{TE}_{\mathrm{Z}}$ waveguide modes as

$$
\begin{aligned}
\mathbf{E}^{b}=\mathbf{E}^{T M}+\mathbf{E}^{T E} & =-j k_{b} Z_{b}\left(\widehat{z} \Psi^{T M}\right)-\nabla \times\left(\widehat{z} \Psi^{T E}\right) \\
\mathbf{H}^{b} & =\mathbf{H}^{T M}+\mathbf{H}^{T E}=\nabla \times\left(\widehat{z} \Psi^{T M}\right)-j k_{b} y_{b}\left(\widehat{z} \Psi^{T E}\right)
\end{aligned}
$$

where $k_{b}=\mathcal{N}_{b} k_{o}$ is the wave number inside the cavity and $Z_{b}=$ $1 / Y_{b}$ is the intrinsic impedance of the filling material. The functions $\Psi^{T M}$ and $\Psi^{T E}$ are the wave potentials both satisfying the scalar wave equation

$$
\left(\frac{\partial^{2}}{\partial x^{2}}+\frac{\partial^{2}}{\partial y^{2}}+k_{b}^{2}\right) \Psi=0
$$

subject to the boundary conditions

$$
E_{y}=E_{z}=0 \quad, \quad x= \pm w
$$

on the cavity side walls, and

$$
\mathbf{M}^{\prime}=\mathbf{E} \times \widehat{n}^{\prime} \quad, \quad y=0
$$

over the aperture. In addition, the potentials must satisfy the impedance boundary condition (2) on the floor and in scalar form

$$
\begin{array}{ll}
H_{z}=v_{f} Y_{b} E_{x} & , \quad y=-d \\
H_{x}=-v_{f} Y_{b} E_{z} & ,
\end{array}
$$

Below, we consider the two principal polarizations separately. 


\section{$\underline{\text { H-polarization }}$}

For $H$-polarization (the $\mathrm{TE}_{\mathrm{Z}}$ case) we have

$$
H_{z}^{i a}=H_{z}^{\mathrm{inc}}+H_{z}^{\mathrm{refl}}=2 e^{j k_{\circ} x \cos \phi_{o}}
$$

which is the geometrical optics field with the aperture closed. The tangential component of the external scattered field is given by

$$
H_{z}^{\alpha}\left(M_{z}\right)=-\frac{k_{o} Y_{o}}{2} \int_{-w}^{w} M_{z}\left(x^{\prime}\right) H_{o}^{(2)}\left(k_{o}\left|x-x^{\prime}\right|\right) d x^{\prime}
$$

while the internal fields are given by (8) and (9) and in this case we have

$$
\begin{aligned}
\mathbf{E}^{b}=\mathbf{E}^{T E} & =-\nabla \times\left(\widehat{z} \Psi^{T E}\right)=-\widehat{x} \frac{\partial}{\partial y} \Psi^{T E}+\widehat{y} \frac{\partial}{\partial x} \Psi^{T E} \\
\mathbf{H}^{b}=\mathbf{H}^{T E} & =-j k_{b} Y_{b}\left(\widehat{z} \Psi^{T E}\right)
\end{aligned}
$$

In order to find useful expressions for $\mathbf{E}^{b}$ and $\mathbf{H}^{b}$, we need to solve for the wave potential $\Psi^{T E}$. To this end, $\Psi^{T E}$ can be expressed as an infinite sum of orthogonal modes

$$
\Psi^{T E}=\sum_{p=0}^{\infty} A_{p} \psi_{p}^{h}
$$

where $\psi_{p}^{h}$ are the waveguide modes all satisfying the wave equation (10) and $A_{p}$ are coefficients to be found. Substituting for $\Psi^{T E}$ in (17), and using (11) and (13), the boundary conditions to be imposed are

$$
\begin{aligned}
& \frac{\partial}{\partial x} \psi_{p}^{h}=0 \quad, \quad x= \pm w \\
& \frac{\partial}{\partial y} \psi_{p}^{h}=j k_{b} \eta_{f} \psi_{p}^{h} \quad, \quad y=-d
\end{aligned}
$$

where $\eta_{f}=1 / v_{f}$ is the normalized impedance of the floor. A set of eigenfunctions satisfying the first condition and the wave equation is

$$
\psi_{p}^{h}=\left[e^{j k_{p}(y+d)}+R_{h} e^{-j k_{p}(y+d)}\right] \cos \left[\frac{p \pi}{2 w}(x-w)\right]
$$


where $R_{h}$ is the reflection coefficient of the floor and $k_{p}$ satisfies the separation parameter equation

$$
k_{p}^{2}=k_{b}^{2}-\left(\frac{p \pi}{2 w}\right)^{2}
$$

Enforcing the second condition (21) and solving for $R_{h}$ gives

$$
R_{h}=\frac{v_{f}-\frac{k_{b}}{k_{p}}}{v_{f}+\frac{k_{b}}{k_{p}}}
$$

We now seek to find the coefficients $A_{p}$ by imposing the condition (12) on the aperture. Thus, we have

$$
\sum_{p} j k_{p} A_{p}\left(e^{j k_{p} d}-R_{h} e^{-j k_{p} d}\right) \cos \left[\frac{p \pi}{2 w}(x-w)\right]=-M_{z}^{\prime}=M_{z}
$$

Multiplying both sides by $\cos \left[\frac{q \pi}{2 w}(x-w)\right]$ and integrating over the aperture yields

$$
\begin{aligned}
\sum_{p} j k_{p} A_{p}\left(e^{j k_{p} d}-\right. & \left.R_{h} e^{-j k_{p} d}\right) \int_{-w}^{w} \cos \left[\frac{p \pi}{2 w}(x-w)\right] \cos \left[\frac{q \pi}{2 w}(x-w)\right] d x \\
& =\int_{-w}^{w} M_{z}(x) \cos \left[\frac{q \pi}{2 w}(x-w)\right] d x
\end{aligned}
$$

and by invoking the orthogonality relation

$$
\int_{-w}^{w} \cos \left[\frac{p \pi}{2 w}(x-w)\right] \cos \left[\frac{q \pi}{2 w}(x-w)\right] d x= \begin{cases}w\left[1+\delta_{p o}\right] & , p=q \\ 0 & , p \neq q\end{cases}
$$

we find

$$
\begin{gathered}
A_{p}=\frac{j}{\left[1+\delta_{p o}\right] w k_{p}\left(e^{j k_{p} d}-R_{h} e^{-j k_{p} d}\right)} \\
\cdot \int_{-w}^{w} M_{z}(x) \cos \left[\frac{p \pi}{2 w}(x-w)\right] d x
\end{gathered}
$$

where $\delta_{p q}$ is the Kronecker delta 


$$
\delta_{p q}= \begin{cases}1, & p=q \\ 0, & p \neq q\end{cases}
$$

The function $\Psi^{T E}$ in (19) is thus completely defined and the tangential magnetic field just below the aperture may be expressed as

$$
H_{z}^{b}\left(-M_{z}\right)=-j k_{b} Y_{b} \int_{-w}^{w} M_{z}\left(x^{\prime}\right) G^{h}\left(x ; x^{\prime}\right) d x^{\prime}
$$

where the Green's function is given by

$$
\begin{gathered}
G^{h}\left(x ; x^{\prime}\right)=\sum_{p=0}^{\infty} \frac{j \varepsilon_{p}}{w k_{p}}\left[\frac{e^{j k_{p} d}+R_{h} e^{-j k_{p} d}}{e^{j k_{p} d}-R_{h} e^{-j k_{p} d}}\right] \\
\cdot \cos \left[\frac{p \pi}{2 w}(x-w)\right] \cos \left[\frac{p \pi}{2 w}\left(x^{\prime}-w\right)\right]
\end{gathered}
$$

and

$$
\varepsilon_{p}=\frac{1}{1+\delta_{p o}}
$$

Substituting (15), (16), and (29) into (5) we obtain

$$
\begin{array}{r}
Z_{o} e^{j k_{o} x \cos \phi_{o}}=\frac{k_{o}}{4} \int_{-w}^{w} M_{z}\left(x^{\prime}\right) H_{o}^{(2)}\left(k_{o}\left|x-x^{\prime}\right|\right) d x \\
+\sum_{p=0}^{\infty} \varepsilon_{p} v_{h p} \cos \left[\frac{p \pi}{2 w}(x-w)\right] \int_{-w}^{w} M_{z}\left(x^{\prime}\right) \cos \left[\frac{p \pi}{2 w}\left(x^{\prime}-w\right)\right] d x^{\prime}
\end{array}
$$

where $v_{h p}$ are the normalized $\mathrm{H}$-mode admittances of the cavity given by

and

$$
v_{h p}=y_{b} \frac{v_{f}+j \frac{k_{b}}{k_{p}} \tan k_{p} d}{j v_{f} \frac{k_{p}}{k_{b}} \tan k_{p} d+1}
$$

$$
y_{b}=\frac{1}{2 w}\left(Y_{b} / Y_{o}\right)
$$


is the normalized characteristic admittance of the material in the groove. Equation (32) is an exact integral equation to be solved for $M_{z}(x)$.

\section{E-polarization}

For E-polarization (the $\mathrm{TM}_{Z}$ case), we have

$$
H_{x}^{i \alpha}=-2 Y_{o} \sin \phi_{o} e^{j k_{o} x \cos \phi_{o}}
$$

and the corresponding tangential scattered fields are given by

$$
H_{x}^{a}\left(M_{x}\right)=\frac{k_{o} Y_{o}}{2}\left(1+\frac{1}{k_{o}^{2}} \frac{\partial^{2}}{\partial x^{2}}\right) \int_{-w}^{w} M_{x}\left(x^{\prime}\right) H_{o}^{(2)}\left(k_{o}\left|x-x^{\prime}\right|\right) d x^{\prime}
$$

and

$$
H_{x}^{b}\left(-M_{x}\right)=-j \frac{Y_{b}}{k_{b}} \int_{-w}^{w} M_{x}\left(x^{\prime}\right) G^{e}\left(x ; x^{\prime}\right) d x^{\prime}
$$

To find the Green's function $G^{e}$ we note that

$$
\begin{aligned}
\mathbf{E}^{b} & =\mathbf{E}^{T M}=-j k_{b} Z_{b}\left(\widehat{z} \Psi^{T M}\right) \\
\mathbf{H}^{b}=\mathbf{H}^{T M} & =\nabla \times\left(\widehat{z} \Psi^{T M}\right)=\hat{x} \frac{\partial}{\partial y} \Psi^{T M}-\widehat{y} \frac{\partial}{\partial x} \Psi^{T M}
\end{aligned}
$$

Following steps similar to those taken for the H-polarization case, the wave potential $\Psi^{T M}$ is expressed as

$$
\Psi^{T M}=\sum_{p=0}^{\infty} B_{p} \psi_{p}^{e}
$$

and the boundary conditions to be satisfied are

$$
\begin{aligned}
& \psi_{p}^{e}=0 \quad, \quad x= \pm w \\
& \psi_{p}^{e}=j k_{b} \eta_{f} \frac{\partial}{\partial y} \psi_{p}^{e} \quad, \quad y=-d
\end{aligned}
$$

suggesting the following form for the eigenfunctions $\psi_{p}^{e}$

$$
\psi_{p}^{e}=\left[e^{j k_{p}(y+d)}-R_{e} e^{-j k_{p}(y+d)}\right] \sin \left[\frac{p \pi}{2 w}(x-w)\right]
$$


where $R_{e}$ is the reflection coefficient of the floor given by

$$
R_{e}=\frac{v_{f}-\frac{k_{p}}{k_{b}}}{v_{f}+\frac{k_{p}}{k_{b}}}
$$

and $k_{p}$ is defined in (23). Enforcing now the boundary condition (12) on the aperture, we have

$$
\sum_{p} k_{p} B_{p}\left(e^{j k_{p} d}-R_{e} e^{-j k_{p} d}\right) \sin \left[\frac{p \pi}{2 w}(x-w)\right]=M_{x}
$$

To find $B_{p}$, we multiply both sides by $\sin \left[\frac{q \pi}{2 w}(x-w)\right]$ and integrate over the extent of the aperture. As before, by employing the orthogonality relation

$$
\int_{-w}^{w} \sin \left[\frac{p \pi}{2 w}(x-w)\right] \sin \left[\frac{q \pi}{2 w}(x-w)\right] d x= \begin{cases}w\left[1-\delta_{p o}\right] & , p=q \\ 0 & , p \neq q\end{cases}
$$

we find

$$
B_{p}=-\frac{Y_{b}}{j k_{b} w\left(e^{j k_{p} d}-R_{e} e^{-j k_{p} d}\right)} \int_{-w}^{w} M_{x}(x) \sin \left[\frac{p \pi}{2 w}(x-w)\right] d x
$$

The tangential magnetic field in the internal region may now be expressed explicitly as

$$
\begin{aligned}
H_{x}(x, 0)= & j \frac{Y_{b}}{k_{b}} \sum_{p=1}^{\infty} \frac{k_{p}}{w}\left[\frac{e^{j k_{p} d}+R_{e} e^{-j k_{p} d}}{e^{j k_{p} d}-R_{e} e^{-j k_{p} d}}\right] \sin \left[\frac{p \pi}{2 w}(x-w)\right] \\
& \cdot \int_{-w}^{w} M_{x}\left(x^{\prime}\right) \sin \left[\frac{p \pi}{2 w}\left(x^{\prime}-w\right)\right] d x^{\prime}
\end{aligned}
$$

and comparing this with (37) we deduce that

$$
\begin{gathered}
G^{e}\left(x, x^{\prime}\right)=-\sum_{p=1}^{\infty} \frac{k_{p}}{w}\left[\frac{e^{j k_{p} d}+R_{e} e^{-j k_{p} d}}{e^{j k_{p} d}-R_{e} e^{-j k_{p} d}}\right] \\
\cdot \sin \left[\frac{p \pi}{2 w}(x-w)\right] \sin \left[\frac{p \pi}{2 w}\left(x^{\prime}-w\right)\right]
\end{gathered}
$$

Substituting (35)-(37) and (49) into (5) yields the integral equation 


$$
\begin{aligned}
\sin \phi_{o} e^{j k x \cos \phi_{o}} & =\frac{k_{o}}{4}\left(1+\frac{1}{k_{o}^{2}} \frac{\partial^{2}}{\partial x^{2}}\right) \int_{-w}^{w} M_{x}\left(x^{\prime}\right) H_{o}^{(2)}\left(k_{o}\left|x-x^{\prime}\right|\right) d x^{\prime} \\
& +\sum_{p=1}^{\infty} v_{e p} \sin \left[\frac{p \pi}{2 w}(x-w)\right] \int_{-w}^{w} M_{x}\left(x^{\prime}\right) \sin \left[\frac{p \pi}{2 w}\left(x^{\prime}-w\right)\right] d x^{\prime}(50)
\end{aligned}
$$

where

$$
v_{e p}=y_{b} \frac{v_{f}+j \frac{k_{p}}{k_{b}} \tan k_{p} d}{j v_{f} \frac{k_{b}}{k_{p}} \tan k_{p} d+1}
$$

are the normalized $\mathrm{E}$-mode admittances of the cavity and $y_{b}$ is given by (34). Equation (50) is an exact integral equation to be solved for $M_{x}(x)$.

Clearly, (32) and (50) are both invalid when

$$
\tan \left(k_{p} d\right)=0
$$

and this occurs only when the material filling the groove is lossless. To be specific, the modal solutions fail if there exist integers $p$ and $q$ such that ${ }^{1}$

$$
\left(\frac{p}{2 w}\right)^{2}+\left(\frac{q}{d}\right)^{2}=\left(\frac{2 \mathcal{N}_{b}}{\lambda_{o}}\right)^{2} \quad p, q \in \mathcal{I}
$$

This difficulty in the evaluation of the internal Green's functions may be circumvented by assuming a small loss in the material or slightly disturbing the geometry of the cavity. We also note that for the proper behavior of the field in the internal region, we must have

$$
\Re \mathrm{e}\left\{k_{p}\right\} \geq 0 \quad, \quad \Im \mathrm{m}\left\{k_{p}\right\} \leq 0
$$

when using (23).

The integral equations (32) and (50) are solved numerically by the moment method. The discretization procedure and the expressions for the admittance matrix elements are given in the Appendix. Upon a solution of the integral equations, the scattered field in the half space $z>0$ can be found from the scattering integral. In particular,

${ }^{1}$ The formulation for the H-polarization case also fails if $k_{p}=0$ in addition to (52). This is equivalent to $p / q=2 w / d$ when $p, q \in \mathcal{I}$. 
the radar scattering echo width of the groove is defined as

$$
\sigma=\lim _{\rho \rightarrow \infty} 2 \pi \rho \frac{\left|\mathbf{H}^{\mathrm{a}}\right|^{2}}{\left|\mathbf{H}^{\text {inc }}\right|^{2}}
$$

and therefore

$$
\sigma_{h}=k_{o}\left|Y_{o} \int_{-w}^{w} M_{z}\left(x^{\prime}\right) e^{j k_{o} x^{\prime} \cos \phi} d x^{\prime}\right|^{2}
$$

and

$$
\sigma_{e}=k_{o}\left|\sin \phi \int_{-w}^{w} M_{x}\left(x^{\prime}\right) e^{j k_{o} x^{\prime} \cos \phi} d x^{\prime}\right|^{2}
$$

for H- and E-polarizations, respectively. If-as is physically the casethe groove is long but finite in length, the physical optics approximation may be used to relate the three-dimensional radar cross section to the echo width (55) calculated on the assumption of infinite length. Hence, for plane wave illumination normal to the $z$-axis, we have

$$
\sigma^{3 d}=2\left(\frac{\ell}{\lambda_{o}}\right)^{2} \sigma
$$

where $\ell$ denotes the actual length of the groove.

\section{Unifromly filled Grooves}

For a homogeneously filled or empty groove terminated by a perfect conductor, we set $\eta_{f}=0$ and find

$$
v_{h p}=-j y_{b} \frac{k_{b}}{k_{p}} \cot k_{p} d \quad v_{e p}=-j y_{b} \frac{k_{p}}{k_{b}} \cot k_{p} d
$$

Figure 3 shows a sample calculation of the backscattering echo width for a $10 \lambda_{0}$ long empty groove based on the above formulation. The results are in good agreement with a corresponding finite element method (FEM) solution [11].

The echo width of a $1 \lambda_{o}$ wide groove filled with a nonmagnetic material of dielectric constant $\epsilon_{b}^{\prime}=4$ at normal incidence is shown in Figure 4 as a function of its depth. The two curves correspond to two different values of the loss tangent $\epsilon_{b}^{\prime \prime} / \epsilon_{b}^{\prime}$. The apparent damping 

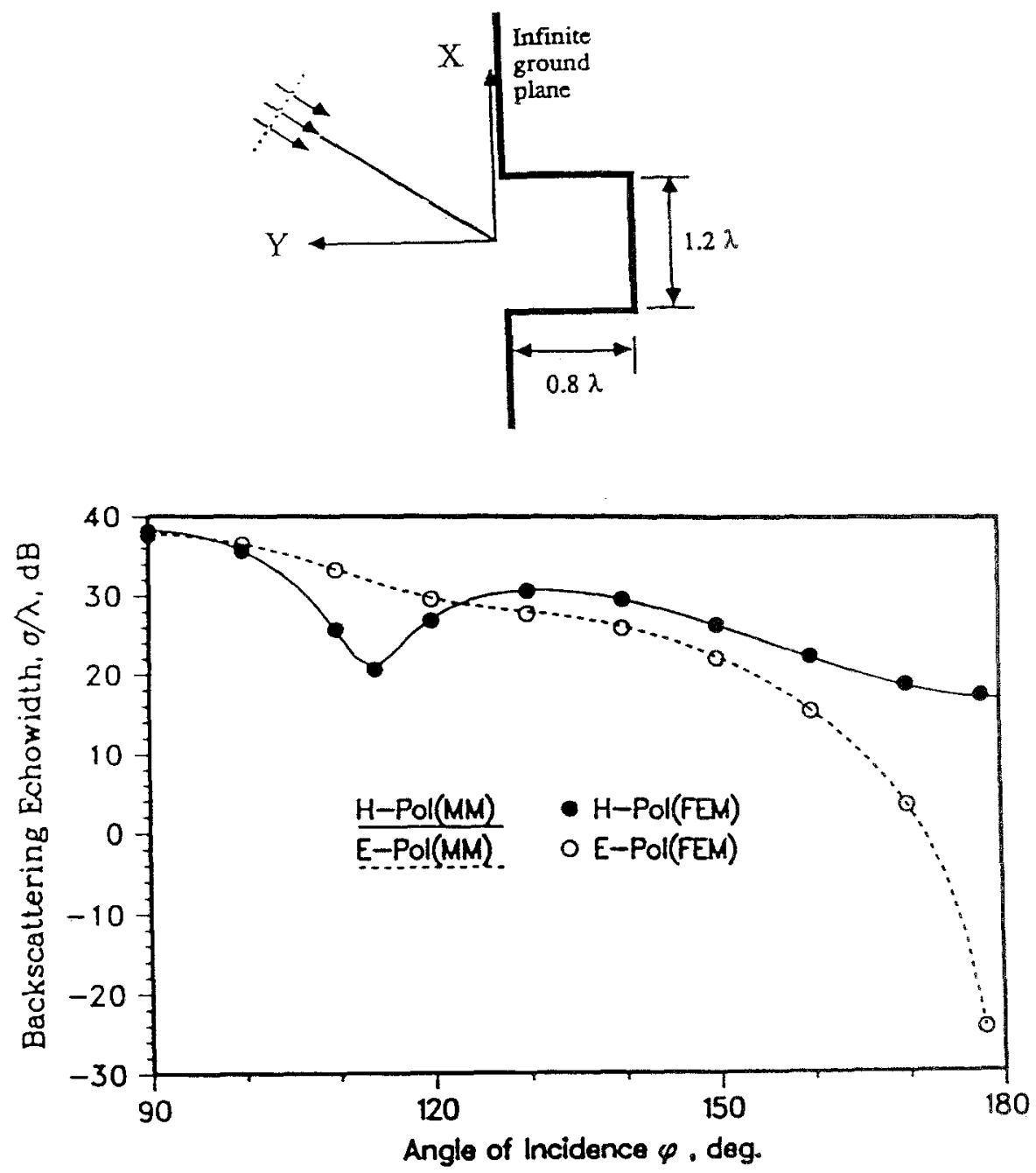

Figure 3: Comparison of the backscattering patterns of a long two-dimensional groove obtained from a finite element solution (FEM) [11] and the method of moments (this study). The groove is assumed to be $10 \lambda$ long. ( 20 samples/ $\lambda$ with 60 waveguide modes). 


\section{Echowidth of a Uniformly Filled Groove}

\section{Normal Incidence, $\mathrm{H}-\mathrm{Pol}$.}

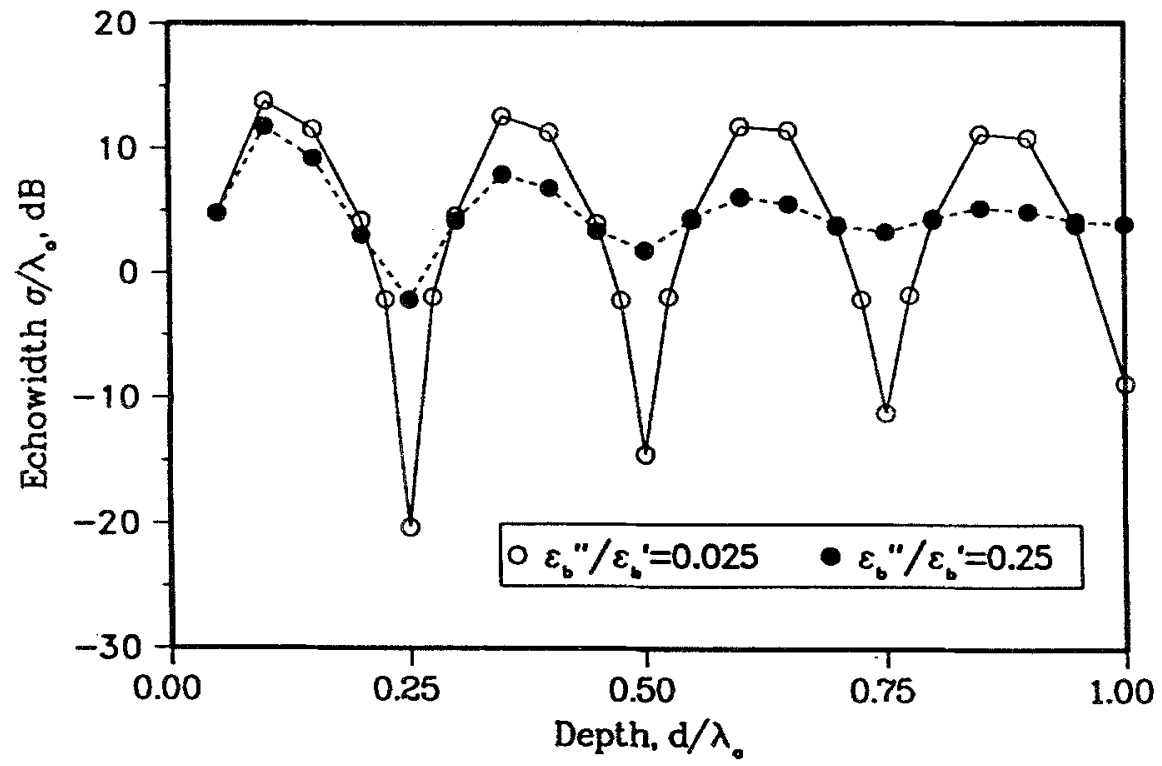

Figure 4: H-polarization echo width of a uniformly filled $1 \lambda_{o}$ wide groove as function of depth at normal incidence. The filling material is nonmagnetic and have $\epsilon_{f}=4-j 0.1(\circ \circ \circ)$ and $\epsilon_{f}=4-j 1.0(\bullet \bullet)$.

effect in the resonance pattern is due to the dielectric losses inside the cavity.

Figure 5 shows the echo width of a $0.5 \lambda_{\circ}$ deep groove uniformly filled with a low-loss material $\left(\epsilon_{b}=2.54-j 0.01\right)$ as a function of its width for both principal polarizations. Also shown are the lowfrequency quasi-static solutions derived in [3]. It is observed that the quasi-static solutions remain valid for up to about $2 w=0.5 \lambda_{\circ}$ and break down for wider apertures in which case the full-wave solution is required. 

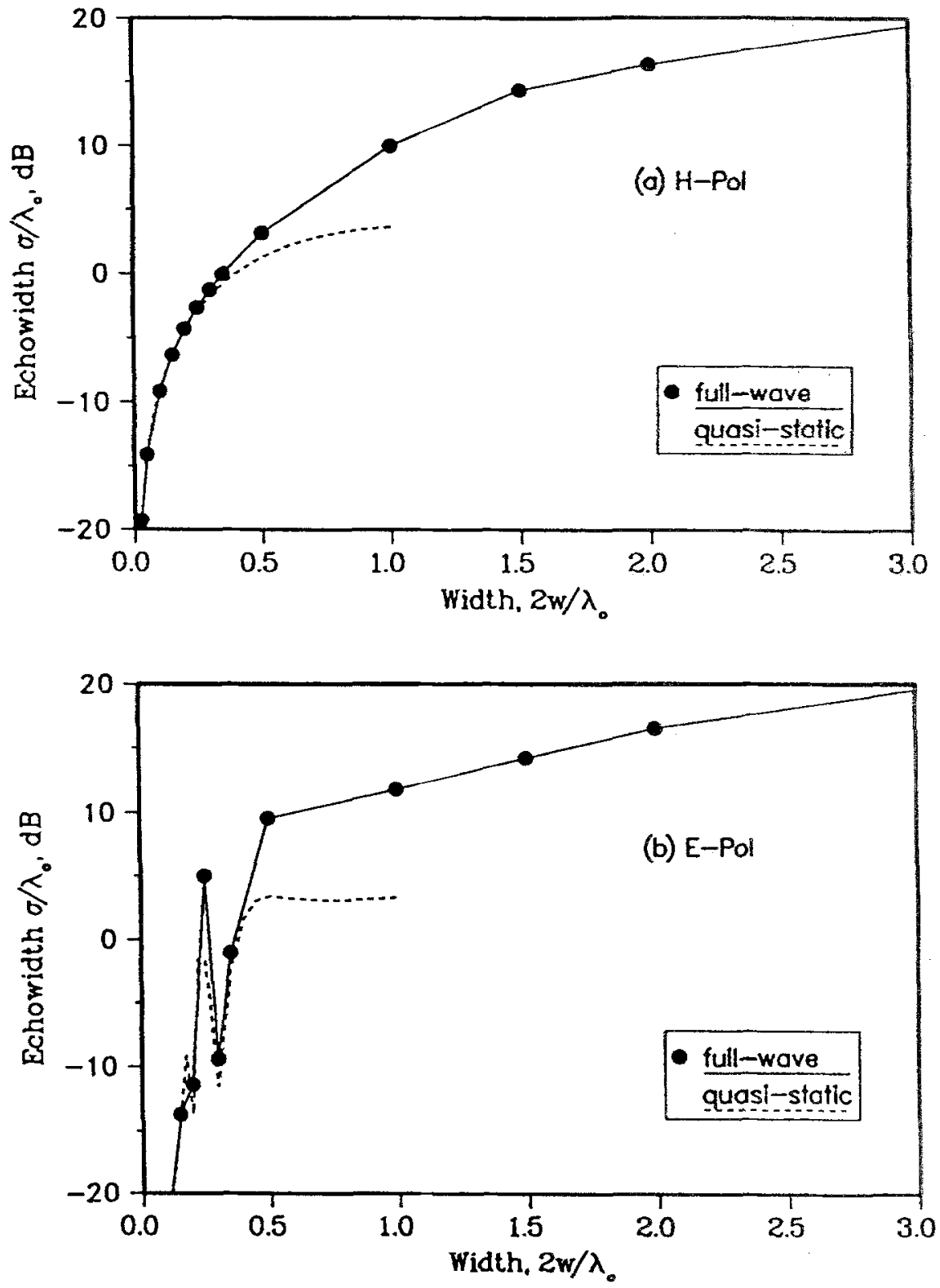

Figure 5: Echo width of a $0.5 \lambda_{0}$ deep groove uniformly filled with a nonmagnetic material $\left(\epsilon_{b}=2.54-j 0.01\right)$ as a function of width at normal incidence: comparison of the full-wave (-) and the quasi-static solution (- - ) [3] for (a) H-polarization and (b) E-polarization. 


\section{$\underline{\text { Partially Loaded Grooves }}$}

The integral equations (32) and (50) may be implemented for computing the scattering by a partially loaded groove where the lower portion of the groove is filled with a dielectric material $\left(\mathcal{N}_{f}=\sqrt{\epsilon_{f} \mu_{f}}\right)$. In this case, the material fills the interior region $z<-d$, is of thickness $t$, and is composed of a high contrast material (Figure 6a). The top portion of the interior region $-d<z<0$ is empty and thus $k_{b}=k_{o}$. In employing the above formulation, the material was replaced by an impedance sheet at $z=-d$ such that (see eqn. (2))

$$
v_{f}=-j \sqrt{\frac{\epsilon_{f}}{\mu_{f}}} \cot \left(\mathcal{N}_{f} k_{o} t\right)
$$

as shown in Figure 6b. This model remains valid if [7]

$$
\left|\mathcal{N}_{f}\right| \gg 1
$$

and

$$
\delta_{f} / t \ll 1
$$

where $\delta_{f}$ is the skin depth of the material

$$
\delta_{f}=\frac{1}{\left|\Im m\left\{\mathcal{N}_{f}\right\}\right| k_{o}}
$$

The first condition ensures that within the medium, the field behaves essentially as a plane wave propagating in the direction of the inward normal to the filling layer. The second condition, on the other hand, imposes the requirement that the inward traveling field suffers enough attenuation so that no outward traveling waves exit at the interface due to reflection.

The computed E- and H-polarization backscattering patterns are shown in Figure 7 for $t=0.3 \lambda_{o}, d=0.2 \lambda_{o}$, and $w=\lambda_{o}$ with the lossy filling material having relative permittivity $\epsilon_{f}=12.5-j 2.5$ and permeability $\mu_{f}=4.5-j 1.2$. Conditions (61)-(62) are satisfied in this case

$$
\left|\mathcal{N}_{f}\right|=7.7 \quad \delta_{f} / t=0.18
$$



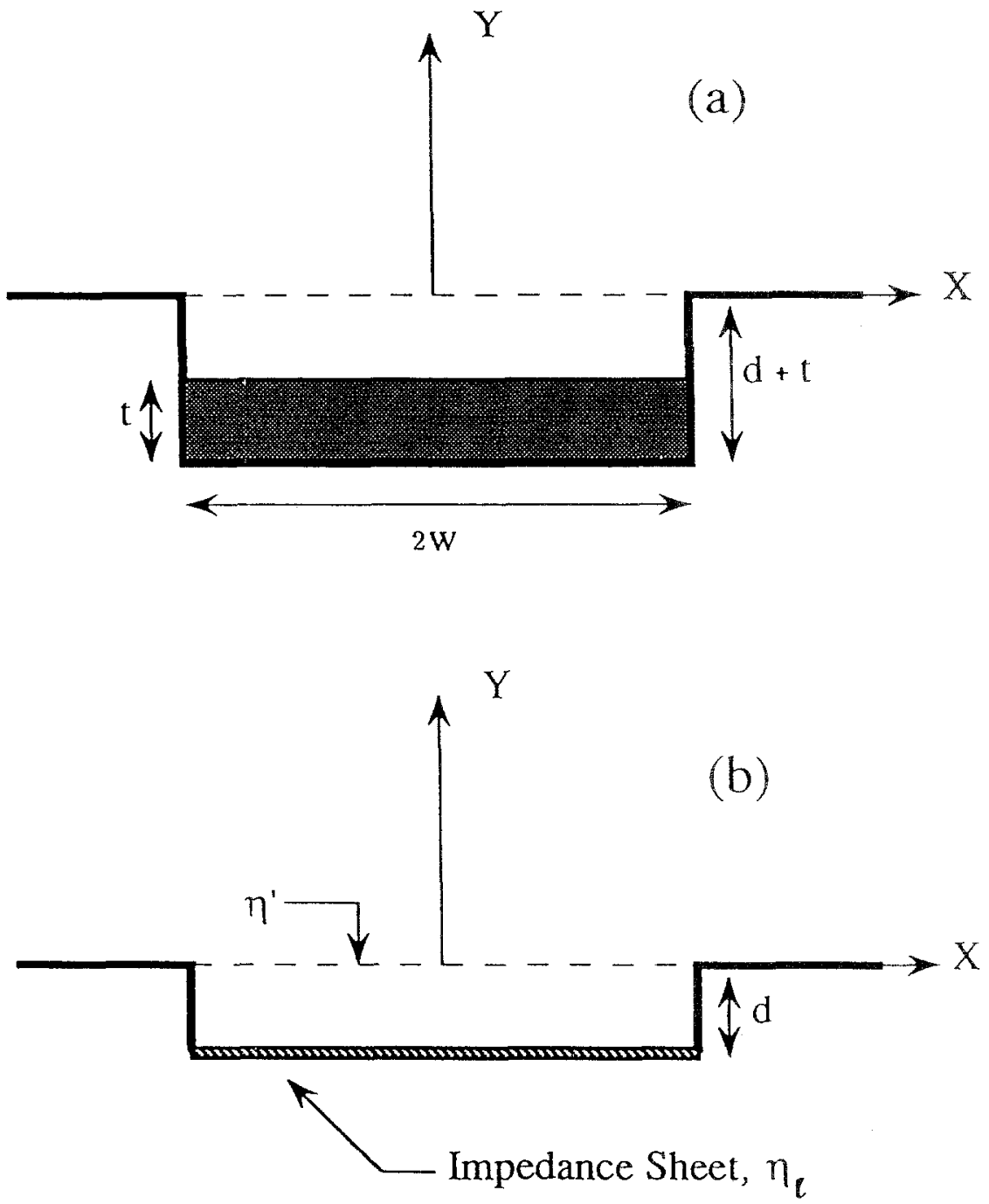

Figure 6: A partially loaded groove with an electrically dense material. (a) Actual geometry. (b) Equivalent problem using an impedance sheet. 


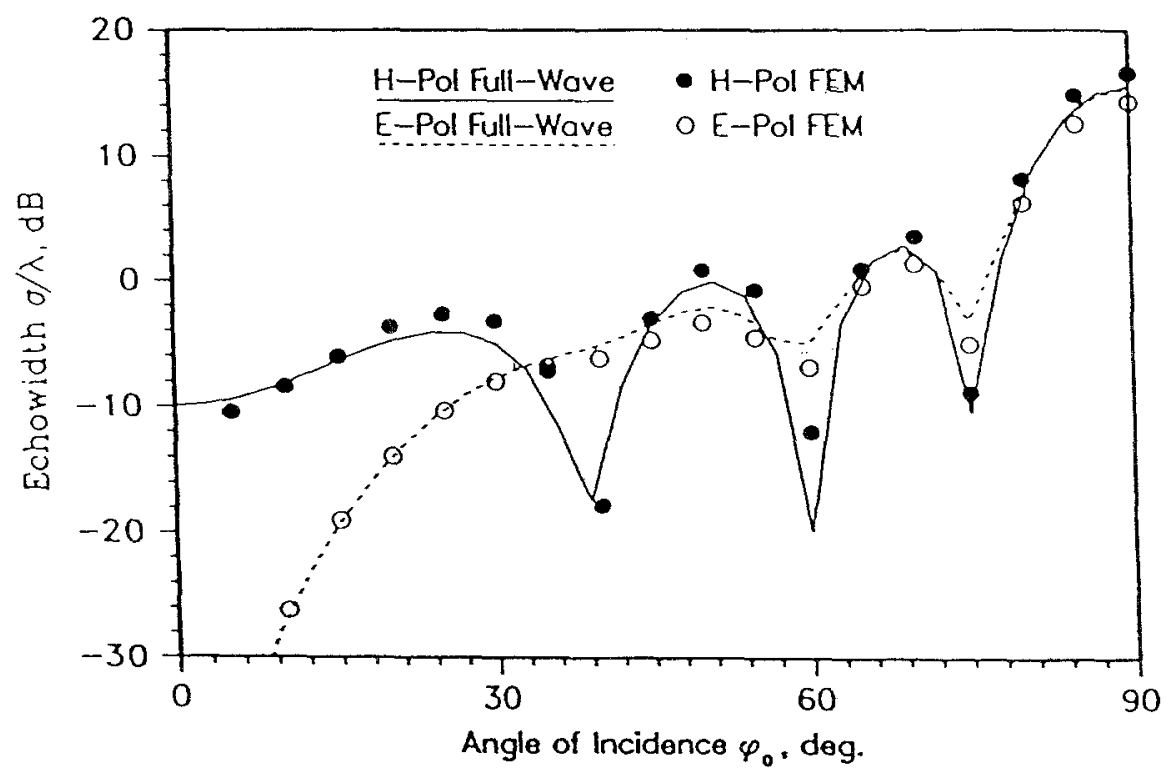

Figure 7: Scattering from the partially loaded groove shown in Figure 6 with $t=0.3 \lambda_{o}, d=0.2 \lambda_{o}$, and $w=\lambda_{o}$. The filling material has $\epsilon_{f}=12.5-j 2.5, \mu_{f}=4.5-j 1.2$ indicating a highly refractive medium $\left(\left|\mathcal{N}_{f}\right|=7.7\right)$ with a relatively small skin depth $\left(\delta_{f} / t=0.18\right)$. 
and as seen, the results are in good agreement with the corresponding data based on the finite element-boundary integral method $[12,13]$ used to simulate the original groove geometry without invoking the impedance boundary condition.

\section{$\underline{\text { Densely Packed Grooves }}$}

When the groove is perfectly loaded with an electrically dense material, it may be efficiently modeled as an impedance insert in a ground plane $[8,9]$. The integral equations (32) and (50) in this case reduce to those corresponding to a direct application of the standard impedance boundary condition (2) over the aperture. In particular, by letting $d \rightarrow 0$ in Figure 6 corresponding to the completely filled groove, we have

$$
v_{h p}=v_{e p} \rightarrow \frac{v_{f}}{2 w}
$$

and the second members of the integral equations (32) and (50) become

$$
\begin{aligned}
& \frac{v_{f}}{2 w} \int_{-w}^{w} M_{z}\left(x^{\prime}\right)\left[\sum_{p=0}^{\infty} \varepsilon_{p} \cos \left[\frac{p \pi}{2 w}(x-w)\right] \cos \left[\frac{p \pi}{2 w}\left(x^{\prime}-w\right)\right]\right] d x^{\prime} \\
& \frac{v_{f}}{2 w} \int_{-w}^{w} M_{x}\left(x^{\prime}\right)\left[\sum_{p=1}^{\infty} \sin \left[\frac{p \pi}{2 w}(x-w)\right] \sin \left[\frac{p \pi}{2 w}\left(x^{\prime}-w\right)\right]\right] d x^{\prime}
\end{aligned}
$$

where $\frac{v_{f}}{2 w}$ is the normalized characteristic admittance of the impedance insert and we have interchanged the order of summation and integration. Identifying now, the bracketed summations as Dirac delta functions [14]

$$
\begin{aligned}
\sum_{p=0}^{\infty} \cos \frac{p \pi}{2 w} \xi \cos \frac{p \pi}{2 w} \xi^{\prime} & =w \delta\left(\xi-\xi^{\prime}\right) \\
\sum_{p=1}^{\infty} \sin \frac{p \pi}{2 w} \xi \sin \frac{p \pi}{2 w} \xi^{\prime} & =w \delta\left(\xi-\xi^{\prime}\right)
\end{aligned}
$$

we arrive at the well known integral equations based on the impedance boundary condition 


$$
Z_{o} e^{j k_{o} x \cos \phi_{o}}=\frac{v_{f}}{2} M_{z}(x)+\frac{k_{o}}{4} \int_{-w}^{w} M_{z}\left(x^{\prime}\right) H_{o}^{(2)}\left(k_{o}\left|x-x^{\prime}\right|\right) d x^{\prime}
$$

for $\mathrm{H}$-polarization and

$$
\begin{aligned}
& \sin \phi_{o} e^{j k_{o} x \cos \phi_{o}}=\frac{v_{f}}{2} M_{x}(x) \\
&+\frac{k_{o}}{4}\left(1+\frac{1}{k_{o}^{2}} \frac{\partial^{2}}{\partial x^{2}}\right) \int_{-w}^{w} M_{x}\left(x^{\prime}\right) H_{o}^{(2)}\left(k_{o}\left|x-x^{\prime}\right|\right) d x^{\prime}
\end{aligned}
$$

for E-polarization.

The above integral equations have the advantage of avoiding the summations corresponding to the calculation of the cavity Green's functions, hence simplifying the computations significantly. They can be solved by a numerical procedure such as the moment method or the conjugate gradient FFT method. Figure 8 shows the amplitude and phase of the equivalent magnetic current density, as well as bistatic and backscattering echo widths for the groove considered in Figure 7 completely filled with the same lossy material of high index of refraction. As expected, the agreement with the full-wave solution is very good.

Finally, the bistatic echo widths of a $2 \lambda_{o}$ wide, $1 \lambda_{o}$ deep groove at $\phi_{o}=45^{\circ}$ are shown in Figure 9 as the groove is progressively filled with a lossy material. The total depth of the groove $(d+t$ in Figure 6 ) is kept constant. The layer thickness $t$, and relative skin depth $\delta_{f} / t$ are also listed in the figure for each case.

\section{Conclusions}

The problem of scattering from two-dimensional rectangular grooves was studied using a full-wave analysis. The analysis is applicable to grooves of arbitrary width and thickness with perfect or imperfect terminations. The effect of electrically dense loading was simulated using the standard impedance boundary condition. When the groove is completely filled by a high contrast material, its scattering behavior can be represented by a direct application of the impedance boundary condition over the aperture. 

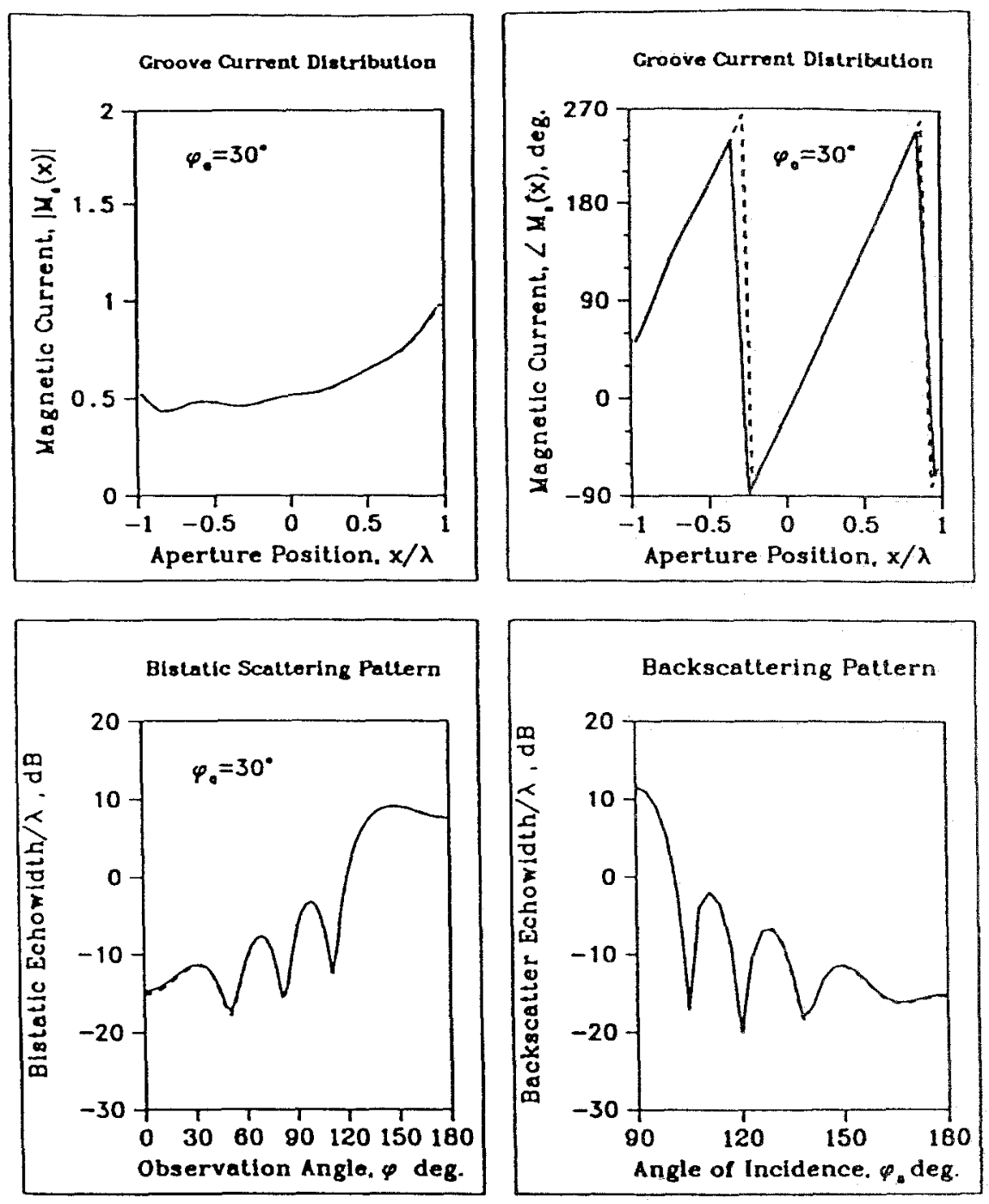

Figure 8: Simulation of a groove filled with a high-contrast material using the standard impedance boundary condition; $w=\lambda_{o}$, $d=0.5 \lambda ; \epsilon_{r}=12.5-j 2.5, \mu_{r}=4.5-j 1.2$, and $\phi_{0}=30^{\circ}$. Comparison of the full-wave (-) and SIBC (- -) results. 
Bistatic Echowidth of a Partially Loaded Groove as a Function of Loading Layer Thickness, E-Pol.

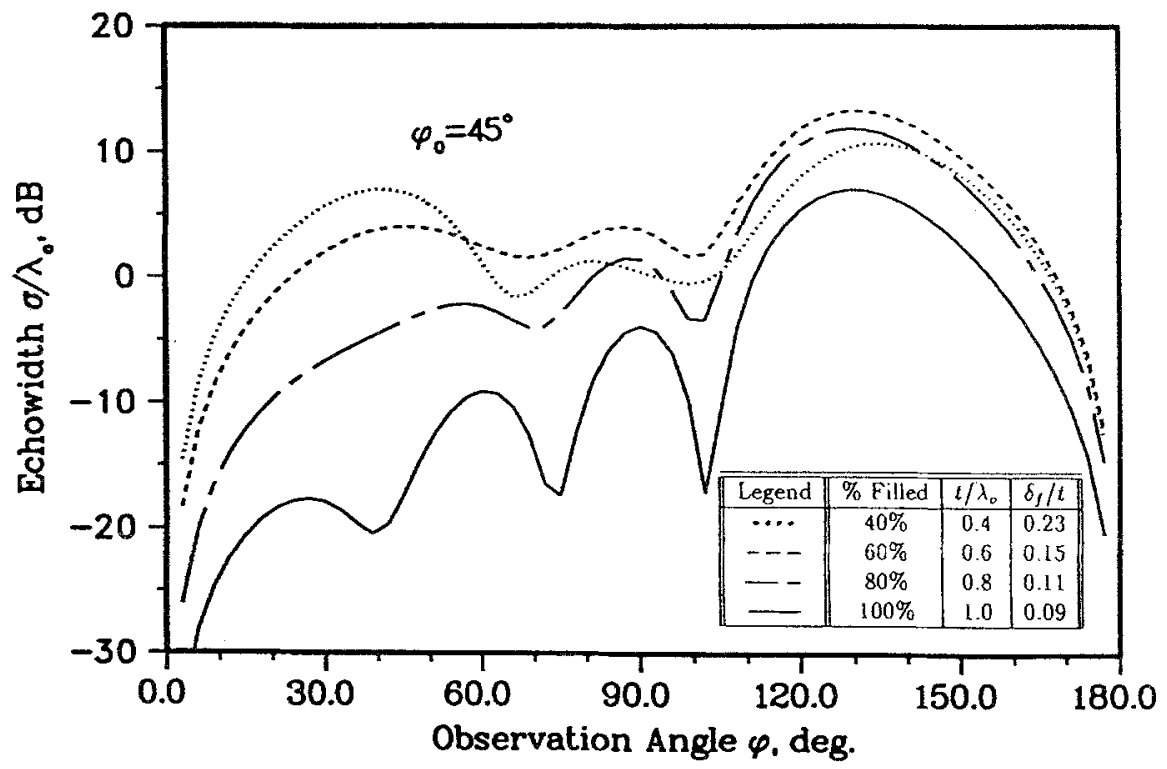

Figure 9: Bistatic echo width of a $2 \lambda_{0}$ wide, $1 \lambda_{0}$ deep groove as it is progressively filled with a dense material $\left(\epsilon_{f}=12.5-\right.$ $\left.j 2.5, \mu_{f}=4.5 .-j 1.2,\left|\mathcal{N}_{f}\right|=7.7\right)$. Incidence angle: $\phi_{o}=45^{\circ}$. 


\section{$\underline{\text { Appendix }}$}

\section{Numerical Discretization}

Considering the H-polarization case, the integral equation (32) may be discretized by expanding $M_{z}(x)$ as

$$
M_{z}(x)=\sum_{n=0}^{N-1} M_{z}\left(x_{n}\right) P\left(x-x_{n}\right), \quad x_{n}=n \Delta+\frac{\Delta}{2}
$$

where $P(x)$ denotes piecewise constant basis function. Substituting for the current expansion in (32) and applying point matching, a system of linear equations is obtained which can be solved easily by standard matrix methods. The elements of the admittance matrix are expressed as

$$
Y_{m n}=\Upsilon_{m n}+\Pi_{m n}
$$

where $\Upsilon_{m n}$ and $\Pi_{m n}$ are elements attributed to the external and internal tangential fields, respectively. They are given by [3]

$$
\Upsilon_{m n} \simeq \begin{cases}\frac{k_{o}}{4} \Delta\left\{1-\frac{j 2}{\pi}\left[\ln \left(\frac{k_{o} \Delta}{4}\right)+\gamma-1\right]\right\}, & n=m \\ \frac{k_{o}}{4} \Delta H_{o}^{(2)}\left(k_{o}\left|x_{m}-x_{n}\right|\right), & n \neq m\end{cases}
$$

and

$$
\Pi_{m n}=\Delta \sum_{p=0}^{\infty} \frac{1}{\varepsilon_{p} 2 w k_{p} \eta_{h p}} \cos \left[\frac{p \pi}{2 w}\left(x_{m}-w\right)\right] \cos \left[\frac{p \pi}{2 w}\left(x_{n}-w\right)\right] \operatorname{sinc}\left(\frac{p \pi}{2 w} \frac{\Delta}{2}\right)
$$

in which $\gamma=1.78108$ is the Euler's constant. A similar discretization can be carried out for E-polarization.

\section{Acknowledgement}

The author is grateful to Professor John L. Volakis for his support and encouragement during the course of this work. The FEM data in Figure 7 were kindly provided by Dr. Jianming Jin. 


\section{References}

[1] R. F. Harrington, "Resonant behavior of a small aperture backed by a conducting body," IEEE Trans. Antenna Propagat., vol. AP-30, no.2, pp. 205-212, Mar. 1982.

[2] T. B. A. Senior and J. L. Volakis, "Scattering by gaps and cracks," IEEE Trans. Antennas Propagat,, vol. AP-37, no. 6, pp. 744-750, June 1989.

[3] K. Barkeshli and J. L. Volakis, "Scattering from narrow rectangular filled grooves," IEEE Trans. Antennas Propagat., vol. AP-39, no. 6, pp. 804-810, June 1991.

[4] R. F. Harrington and J. R. Mautz, "A generalized network formulation for aperture problems," IEEE Trans. Antennas Propagat., vol. AP-24, pp. 870-873, Nov. 1976.

[5] D. T. Auckland and R. F. Harrington, "Electromagnetic transmission through a filled slit in a conducting plate of finite thickness, TE case," IEEE Trans. Microwave Theory Tech,, vol. MTT-26, no. 7, pp. 499-505, Jul. 1978.

[6] S. N. Sinha, D. K. Mehra and R. P. Agarwal, "Radiation from a waveguide-backed aperture in an infinite ground plane in the presence of a thin conducting plate," IEEE Trans. Antennas Propagat., vol. AP-34, no. 4, pp. 539-545, Apr. 1986.

[7] T. B. A. Senior, "Impedance boundary conditions for imperfectly conducting surfaces", Appl. Sci. Res., sec. B, vol. 8, pp. 418-436, 1960.

[8] K. W. Whites and R. Mittra, "A systematic study of the impedance boundary condition", IEEE AP-S International Symposium, Digest pp. 870-873, Dallas, TX, 1990.

[9] K. Barkeshli and J.L. Volakis, "TE Scattering by a twodimensional groove in a ground plane using higher order impedance boundary conditions," IEEE Trans. Antennas Propagat., vol. AP-38, no. 9, pp. 1421-1428, Sept. 1990.

[10] R. F. Harrington, Time Harmonic Electromagnetic Fields, Mc- 
Graw Hill, New York, 1969.

[11] S. W. Lee and H. Ling, Data book for cavity RCS, University of Illinois Electromagnetic Laboratory Technical Report SWL89-1, Jan. 1989.

[12] J.-M. Jin and J. L. Volakis, "TM scattering by an inhomogeneously filled aperture in a thick conducting plane," IEE Proceeding-H, vol. 137, pp. 153-159, June 1990.

[13] J.-M. Jin and J. L. Volakis, "TE scattering by an inhomogeneously filled aperture in a thick conducting plane," IEEE Trans. Antenna Propagat., vol. AP-38, pp. 1280-1286, Aug. 1990.

[14] J. P. Keener, Principles of Applied Mathematics: Transformation and Approximation, Addison-Wesley, New York, 1988, p. 289. 